\title{
Motion Compensated Fan-Beam Reconstruction for Nonrigid Transformation
}

\author{
Katsuyuki Taguchi*, Member, IEEE, and Hiroyuki Kudo, Member, IEEE
}

\begin{abstract}
We develop an approximate fan-beam algorithm to reconstruct an object with time-dependent nonrigid transformation such as the heart. The method is in the form of derivative backprojection filtering with compensation of affine transformations on a local basis. Computer simulations showed the proposed method significantly reduces image artifact due to nonrigid motion. Therefore, with very little motion artifact, the proposed method allowed us to reconstruct images from projections over about one motion cycle, resulting in reduced image noise level down to $40 \%$ of the current level.
\end{abstract} tion.

Index Terms-Computed tomography (CT), motion compensa-

\section{INTRODUCTION}

C ARDIOVASCULAR disease remains the leading cause of death in the western world, placing an ever-increasing burden on both private and public health services [1]. The electrocardiogram-gated cardiac X-ray computed tomography (CT) imaging is a promising noninvasive technique for early detection and characterization of various signs of cardiac diseases such as fatty vulnerable soft plaque (atherosclerosis) in coronary arteries, perfusion defect in myocardial, etc., [2]. However, there are two major problems with the current cardiac technique: large radiation dose to patients and insufficient temporal resolution [3]. The typical radiation dose with cardiac CT is $10-15 \mathrm{mSv}$, which is $3-5$ times as large as a standard chest CT scan. The current temporal resolution is merely $80-165 \mathrm{~ms}$ in contrast to the minimum requirement of $10-30 \mathrm{~ms}$ to observe the beating heart motion without motion artifact. The current technique uses the electrocardiogram signals to select projection data acquired in a time window that is placed within a cardiac cycle with relatively slow motion (e.g., middiastole). Images are then reconstructed by neglecting the cardiac motion within the time window, resulting in blurring and artifacts in the reconstructed images. Also, this technique uses only 10\%-30\% of the acquired data-within the cardiac time window-and

Manuscript received January 23, 2008; revised April 22, 2008. This work was supported in part by American Heart Association under Grant 0665431U, in part by Siemens Medical Solutions under Grant JHU-2006-CT-06-01, and in part by the startup fund of the Division of Medical Imaging Physics at the Russell H. Morgan Department of Radiology and Radiological Sciences, the Johns Hopkins University. Asterisk indicates corresponding author.

*K. Taguchi is with the Russell $\mathrm{H}$. Morgan Department of Radiology and Radiological Sciences, Johns Hopkins University School of Medicine, $601 \mathrm{~N}$. Caroline Street, Baltimore MD21287 USA (e-mail: ktaguchi@ jhmi.edu).

H. Kudo is with the University of Tsukuba, Tsukuba, Ibaraki 305-8573, Japan.

Color versions of one or more of the figures in this paper are available online at http://ieeexplore.ieee.org.

Digital Object Identifier 10.1109/TMI.2008.925076 throws away the rest of "off-phase" data, resulting in unnecessary radiation dose to patient if the tube current is not prospectively modulated. Recently, a method to turn off the X-ray for off-phase has been proposed [4]. This method will reduce the dose significantly at the expense of the functional (motion) information. Our approach has a potential to achieve the largest dose reduction while substantially improving the quality of 3-D and 4-D (4-D = 3-D plus time) images.

Therefore, the long-term goal of this research is to develop time resolved, low-dose cardiac CT imaging [3]. Specifically, we will develop an iterative algorithm that estimates the timedependent motion vector field of the heart from the measured projection data and integrates it into the image reconstruction process. The motion will be estimated by maximizing the agreement between the acquired 4-D projection data and the reconstructed time-resolved 4-D images. The quality of the image will be significantly improved since the motion is compensated. In addition, lower tube current could be utilized since all of the acquired data will be used to reconstruct any cardiac phase of interest. We estimate the reduction of radiation dose to the patient will be 50\%-75\% of the current level [3]. A reduction of noise or dose may be achieved by using a nonlinear processing of projection data [5]-[7], which can be combined with the proposed method for further reduction of noise or dose or both.

Toward this long-term goal, one can incorporate a time-dependent motion vector field into a system matrix and solve this 4-D reconstruction problem using a numerical (statistical) algorithm. Gilland et al. developed a method that jointly estimates and compensates for the motion of the object for single photon emission CT [8], [9]. We aim at developing a similar iterative joint method; however, we want to replace the numerical reconstruction method by an analytical method as it could provide a faster convergence in the iteration process due to its linear nature. In this study, we will develop a method to reconstruct time-resolved 2-D images of dynamic objects from fan-beam projections assuming the time-dependent 2-D motion vector field is known. The algorithm will be integrated into the iterative process later.

Cardiac motion estimation is indeed a very challenging illposed problem. There have been many methods proposed and tested with clinical cases using optimization methods, optical flow methods, and continuum theory [10]-[16].

For respiratory motion correction in lung CT, Crawford's approximate fan-beam algorithm [17] compensates a global expansion/translation of the object. The algorithm was a ramp filtering (FBP) with a change of variables to take a global motion model into account. Roux et al. [18] developed an exact fan-beam algorithm for a global time-dependent affine transformation by incorporating transformation into Noo's derivative filtered backprojection with Hilbert transform kernel (DFBP) 
algorithm [19]. Recently, Desbat et al. extended Roux's motion model; his algorithm allows any deformations that maintain acquisition rays as straight lines [20], [21].

Most of nonrigid transforms such as cardiac or respiratory motion do not satisfy this restriction-lines become curves with deformations. For respiratory motion compensation, Ritchie et al. [22] applied Crawford's algorithm on a local basis by changing the motion model for each pixel. Despite its global nature of the ramp filtering, the reconstructed images were in good quality demonstrating significantly reduced motion artifact. This shows the potential of the correction based on locally accurate motion model, filtering, and backprojection.

We hypothesize that by applying the correction locally, we can approximately reconstruct cardiac images with an acceptable level of error. Our approach to cardiac reconstruction is similar to Ritchie's with an effort to make filtering process much more accurate. We incorporate an affine transformation into a derivative backprojection filtering (DBPF) algorithm [23], [24].

The structure of this paper is as follow. In Section II, we discuss a rigid motion and derive a basic algorithm, DABPF. In Section III, we outline DAxBPF, i.e., how to apply DABPF on a local basis to compensate for nonrigid motion. Sections IV and $\mathrm{V}$ present schemes and results of computer simulations, respectively, followed by discussion and conclusion in Section V.

\section{RigiD Motion}

In this section, we will derive an exact algorithm, DABPF, from DBPF algorithm to reconstruct a dynamic object with globally defined time-dependent affine transformation.

\section{A. Notations}

We use the right hand coordinate system with $\underline{x}=(x, y)$. The time-dependent 2-D deforming object is defined by $f_{0}\left(\underline{x}_{0}\right)$, $\underline{x}_{0} \in \mathrm{R}^{2}$, at the reference time $t=t_{0}$, and $f_{t}\left(\underline{x}_{t}\right), \underline{x}_{t} \in \mathrm{R}^{2}$, at time $t$. The time-dependent affine operation $\Gamma_{t}$ projects a point $\underline{x}_{t}$ at time $t$ to a point $\underline{x}_{0}$ at time $t_{0}$

$$
\Gamma_{t}\left(\underline{x}_{t}\right)=A_{t} \underline{x}_{t}+\underline{B}_{t}=\underline{x}_{0}
$$

where

$$
A_{t}=\left[\begin{array}{ll}
a_{11}(t) & a_{12}(t) \\
a_{21}(t) & a_{22}(t)
\end{array}\right] \text { and } \underline{B}_{t}=\left[\begin{array}{l}
B_{1}(t) \\
B_{2}(t)
\end{array}\right] .
$$

We suppose that this affine deformation is invertible, that is, $\forall t$, $\operatorname{det} A_{t} \neq 0$, where det $A_{t}$ is the determinant of matrix $A_{t}$. The object can thus be written as

$$
f_{t}\left(\underline{x}_{t}\right)=f_{0}\left(\Gamma_{t}\left(\underline{x}_{t}\right)\right)=f_{0}\left(\underline{x}_{0}\right) .
$$

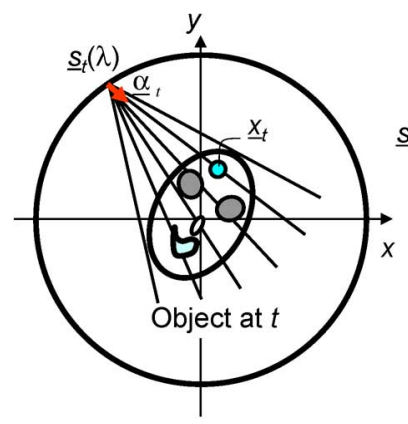

True source trajectory

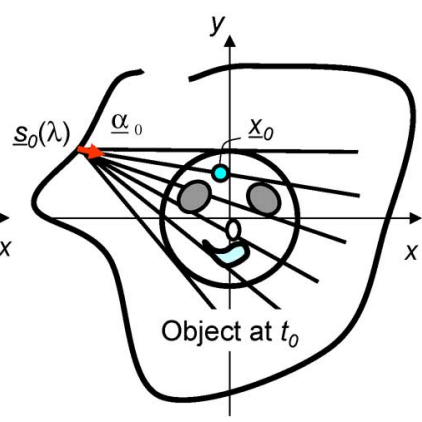

Virtual source trajectory
Fig. 1. Example of true and virtual source trajectories with rigid motion. Straight lines remained as lines after being transformed from time $t$ to $t_{0}$.

The true circular source trajectory $\underline{s}_{t}(\lambda)$ and fan-beam projections $g_{t}\left(\lambda, \underline{\alpha}_{t}\right)$ can be noted as

$$
\begin{aligned}
\underline{s}_{t}(\lambda) & =\left(-R_{s} \cos \lambda,-R_{s} \sin \lambda\right)^{T} \\
g_{t}\left(\lambda, \underline{\alpha}_{t}\right) & =\int_{0}^{\infty} f_{t}\left(\underline{s}_{t}(\lambda)+l \underline{\alpha}_{t}\right) d l
\end{aligned}
$$

where $\lambda \in \mathrm{R}$ is a parameter of the source trajectory, $\underline{\alpha}_{t}$ is a unit vector along a ray from $\underline{s}_{t}(\lambda)$, and $R_{s}$ is the distance from the source to the rotation axis. Note $\lambda$ is monotonically increasing function of time, e.g.,

$$
\lambda=\omega t .
$$

\section{B. DABPF Algorithm}

Let a 2-D function $b_{0, \underline{\theta}}\left(\underline{x}_{0}\right)$ be a Hilbert transformed 2-D image $f_{0}\left(\underline{x}_{0}\right)$ at the reference time $t_{0}$, where $\underline{\theta}$ is a unit vector that defines the direction of 1-D finite inverse Hilbert transform [23] (see also Appendix). We want to first obtain $b_{0, \underline{\theta}}\left(\underline{x}_{0}\right)$ from measured projections $g_{t}\left(\lambda, \underline{\alpha}_{t}\right)$. We transform a circular source orbit $\underline{s}_{t}(\lambda)$ around the object $f_{t}$ into a virtual source trajectory $\underline{s}_{0}(\lambda)$ around the object $f_{0}$ at $t_{0}$ (Fig. 1)

$$
\underline{s}_{0}(\lambda)=\Gamma_{t}\left(\underline{s}_{t}(\lambda)\right) .
$$

The projection data with the virtual path can then be obtained by

$$
g_{0}\left(\lambda, \underline{\alpha}_{0}\right)=\left\|A_{t} \underline{\alpha}_{t}\right\| g_{t}\left(\lambda, \underline{\alpha}_{t}\right)
$$

where

$$
\underline{\alpha}=\frac{A_{t} \underline{\alpha}_{t}}{\left\|A_{t} \underline{\alpha}_{t}\right\|}
$$


Proof: Using (3) inside the integral of the right-hand side of (5) and the linearity, the object $f$ at time $t$ can be noted by

$$
\begin{aligned}
f_{t}\left(\underline{s}_{t}(\lambda)+l \underline{\alpha}_{t}\right) & =f_{0}\left(\Gamma_{t}\left[\underline{s}_{t}(\lambda)+l \underline{\alpha}_{t}\right]\right) \\
& =f_{0}\left(\Gamma_{t}\left[\underline{s}_{t}(\lambda)\right]+l \times \Gamma_{t}\left[\underline{\alpha}_{t}-\underline{O}_{t}\right]\right) \\
& =f_{0}\left(\Gamma_{t}\left[\underline{s}_{t}(\lambda)\right]+l \times\left(A_{t} \underline{\alpha}_{t}+\underline{B}_{t}-\underline{B}_{t}\right)\right) \\
& =f_{0}\left(\underline{s}_{0}(\lambda)+l A_{t} \underline{\alpha}_{t}\right) .
\end{aligned}
$$

Inserting (10) into (5) and changing a variable, $l=\hat{l} /\left\|A_{t} \underline{\alpha}_{t}\right\|$, we get

$$
\begin{aligned}
g_{t}\left(\lambda, \underline{\alpha}_{t}\right) & =\int_{0}^{\infty} f_{0}\left(\underline{s}_{0}(\lambda)+l A_{t} \underline{\alpha}_{t}\right) d l \\
& =\frac{1}{\left\|A_{t} \underline{\alpha}_{t}\right\|} \int_{0}^{\infty} f_{0}\left(\underline{s}_{0}(\lambda)+\hat{l} \frac{A_{t} \underline{\alpha}_{t}}{\left\|A_{t} \underline{\alpha}_{t}\right\|}\right) d \hat{l} \\
& =\frac{1}{\left\|A_{t} \underline{\alpha}_{t}\right\|} \times \int_{0}^{\infty} f_{0}\left(\underline{s}_{0}(\lambda)+\hat{l} \underline{\alpha}_{0}\right) d \hat{l} \\
& =\frac{1}{\left\|A_{t} \underline{\alpha}_{t}\right\|} \times g_{0}\left(\lambda, \underline{\alpha}_{0}\right)
\end{aligned}
$$

where $\underline{\alpha}_{0}$ is given by (9).

The derivative of the virtual projection data, (8), can then be calculated by using product rule and (6)

$$
\begin{aligned}
\frac{\partial}{\partial \lambda} g_{0}\left(\lambda, \underline{\alpha}_{0}\right)= & \frac{\partial}{\partial \lambda}\left\|A_{t} \underline{\alpha}_{t}\right\| g_{t}\left(\lambda, \underline{\alpha}_{t}\right) \\
& +\left\|A_{t} \underline{\alpha}_{t}\right\| \frac{\partial}{\partial \lambda} g_{t}\left(\lambda, \underline{\alpha}_{t}\right) \\
= & \frac{1}{\omega}\left\|\frac{\partial A_{t}}{\partial t} \underline{\alpha}_{t}\right\| g_{t}\left(\lambda, \underline{\alpha}_{t}\right) \\
& +\left\|A_{t} \underline{\alpha}_{t}\right\| \frac{\partial}{\partial \lambda} g_{t}\left(\lambda, \underline{\alpha}_{t}\right)
\end{aligned}
$$

where

$$
\frac{\partial}{\partial \lambda} g_{t}\left(\lambda, \underline{\alpha}_{t}\right)=\lim _{\varepsilon \rightarrow 0} \frac{g_{t}\left(\lambda+\varepsilon, \underline{\alpha}_{t}\right)-g_{t}\left(\lambda, \underline{\alpha}_{t}\right)}{\varepsilon} .
$$

The following procedures are the same as DBPF algorithms (see [23] and Appendix). From here, we drop the argument $\lambda$ from vectors $\underline{s}_{0}, \underline{s}_{0}{ }^{\prime}$, and $\underline{n}_{0}$ for more readability. The differentiated projections are backprojected from the virtual source trajectory $\underline{s}_{0}$

$$
b_{0}\left(\underline{x}_{0}\right)=\int_{\lambda_{1}}^{\lambda_{2}} \frac{w\left(\underline{s}_{0} \cdot \underline{n}_{0}, \underline{n}_{0}\right) \cdot \operatorname{sgn}\left(\underline{\theta} \cdot \underline{n}_{0}\right) \frac{\partial}{\partial \lambda} g_{0}\left(\lambda, \underline{\alpha}_{0}\right)}{\left\|\underline{x}_{0}-\underline{s}_{0}\right\|} d \lambda
$$

where

$$
\begin{aligned}
& \underline{n}_{0}=\underline{s}_{0}^{\prime}-\left[\underline{\alpha}_{0} \cdot \underline{s}_{0}^{\prime}\right] \cdot \underline{\alpha}_{0} \\
& \underline{\alpha}_{0}=\frac{\left(\underline{x}_{0}-\underline{s}_{0}\right)}{\left\|\underline{x}_{0}-\underline{s}_{0}\right\|}
\end{aligned}
$$

$\lambda_{1}, \lambda_{2}$ are both ends of the backprojection range, and $w$ for $\lambda_{i}$ is a normalized redundancy weight defined as

$$
\sum_{i=0}^{\infty} w\left(\underline{s}_{0} \cdot \underline{n}_{0}, \underline{n}_{0}\right)=1 .
$$

Finally, we obtain $f_{0}\left(\underline{x}_{0}\right)$

$$
f_{0}\left(\underline{x}_{0}\right)=-\frac{1}{2 \pi} H^{-1}\left(b_{0}\left(\underline{x}_{0}\right)\right)
$$

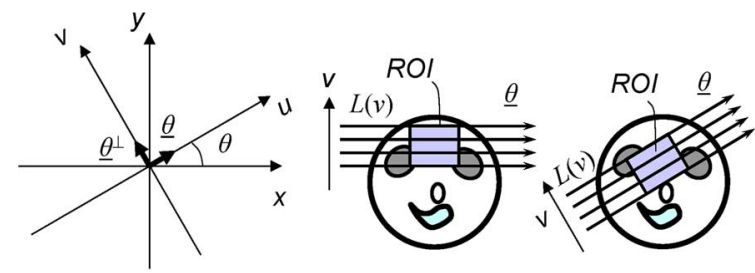

Fig. 2. Two examples of the set of parallel Hilbert filtering lines. Left case does not need step- 4 since $\theta=0$.

where $H^{-1}(\cdot)$ is the 1-D finite inverse Hilbert transform along $\underline{\theta}$ direction [23]. We call this method DABPF algorithm. Note that globally defined affine transformations preserve straight lines as straight lines (see Fig. 1); thus, DABPF is exact and mathematically the same as Roux's DAFBP.

DABPF algorithm starts with measured projections and a known time-dependent globally defined affine transform. We summarize DABPF algorithm by the following four steps that are similar to DBPF algorithm [23], [24]. Consider a $(u, v)$ coordinate system (Fig. 2), rotated by angle $\theta$ from the $(x, y)$-axes. Two coordinate systems are related by $u(x, y)$ and $v(x, y)$.

Step 1) Define a region-of-interest (ROI) and a set of the Hilbert lines $L(v)$ such that

a) every point $\underline{x}=(x, y)$ inside the ROI lies on only one Hilbert line $L(v(x, y))$;

b) all line integral projection data passing through any object point on each Hilbert line are measured.

In Fig. 2, we show two examples on how to choose the set of the Hilbert lines.

Step 2) Along each Hilbert line $L(v(x, y))$, we compute the derivative backprojection by (12)-(14) to obtain the $b_{0}(u, v), 1$-D Hilbert transformed image of $f$ along $\underline{\theta}$.

Step 3) Along each Hilbert line $L(v(x, y))$, we invert the Hilbert transform by using the inversion formula of finite Hilbert transform ([23] and (18)) to obtain a function $(u, v)$, which is the same as $\hat{f}(x, y)$ but is represented on the $(u, v)$ coordinates.

Step 4) From the relation $f(x, y)=\hat{f}(u(x, y), v(x, y))$, we obtain $f(x, y)$. This step is not necessary if $\theta=0$.

\section{Data Sufficiency Condition}

The data sufficiency condition for DABPF is, as a combination of those for DAFBP method [18], [19] and DBPF method [23]: a point $\underline{x}_{0}$ at the reference time $t_{0}$ can be accurately reconstructed from fan-beam projections acquired during a time-dependent affine deformation, provided every line passing through any object point on the filtering line of the point $\underline{x}_{0}$ has an intersection with the virtual source trajectory. Fig. 4 provides a graphical description of the condition.

\section{NONRIGID MOTION}

In this section, we first approximate a nonrigid motion by a spatially varying affine transformation. We will then propose an 


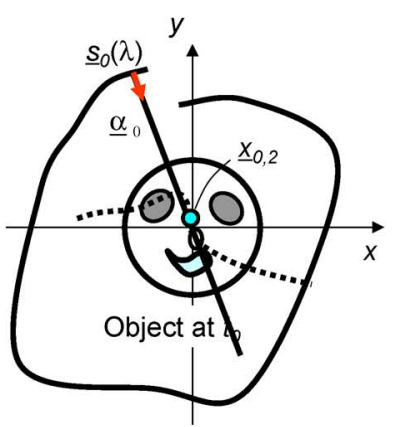

Virtual source trajectory $\underline{s}_{0}(\lambda)$ for $\underline{x}_{0,2}$

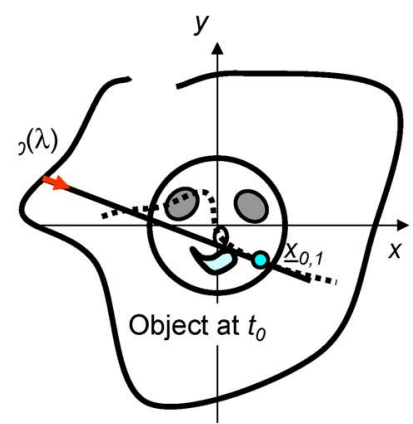

Virtual source trajectory $\underline{s}_{0}(\lambda)$ for $\underline{x}_{0,1}$

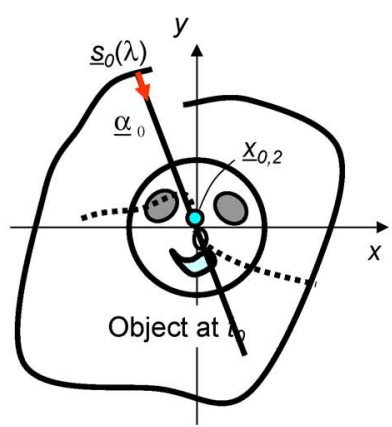

Virtual source trajectory $\underline{s}_{0}(\lambda)$ for $\underline{x}_{0,2}$

Fig. 3. Example of true and virtual source trajectories with nonrigid motion. Straight line at $t$ corresponds to a curve at $t_{0}$. Proposed DAxBPF applies DABPF on a local basis to approximate the curve by its tangent, corresponding $\underline{s}_{0}(\lambda)$, and locally fitted affine transform for each $\underline{x}_{0}$.

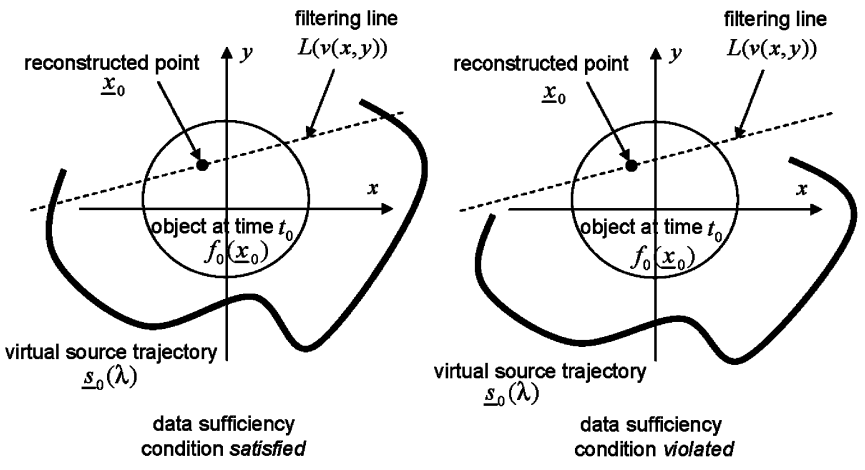

Fig. 4. Point $\underline{x}_{0}$ and a virtual source trajectory which satisfy the data sufficiency conditions described in Sections II-C and III-C (left) and those do not (right).

approximate algorithm, DAxBPF, to reduce the effect of the spatially varying time-dependent affine transformation by applying DABPF on a local basis (see Fig. 3).

\section{A. Spatially Varying Affine Transform}

We consider two scenarios how a nonrigid transformation of the object is given: sparsely defined time-dependent affine transformations; or a time-dependent motion vector field.

The first scenario starts with a finite sets of time-dependent affine transformations $\left.\Gamma_{t k}, \underline{x}_{t, m}\right)$ given only for discrete spatial locations $\underline{x}_{0, m}$ 's, $m=1, \ldots, M$, and temporal samples $t_{k}$ 's, $k=1, \ldots, K$. We then spatially and temporally interpolate each element of matrix and vector of affine transform [see (2)] by, e.g., a cubic spline interpolation, to obtain an affine transformation for each small region-of-interest (ROI) at time $t$ (that corresponds to each projection data). An example is shown in Fig. 5. We focus on this scenario in this paper.

The second scenario starts with a time dependent motion vector field. The time dependent deformation $\underline{M}_{t}\left(\underline{x}_{t}\right)$, defined by a time-dependent motion vector for each location, projects a point $\underline{x}_{t}$ at time $t$ to a point $\underline{x}_{0}$ at time $t_{0}$. The deformation $\underline{M}_{t}\left(\underline{x}_{t}\right)$ has to be bijective (one-to-one and onto) to be invertible. For a small ROI centering $\underline{x}_{t}$, a vector field within the ROI can then be approximated by an affine transformation. Applying it for each $\underline{x}_{t}$ will generate spatially varying affine

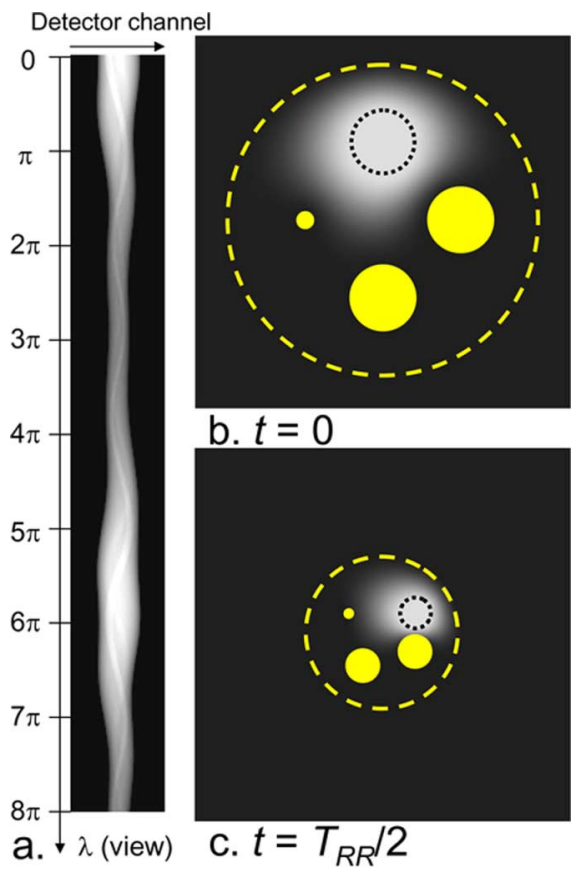

Fig. 5. a: Sinogram of 5-ball phantom with $63 \mathrm{bpm}$. b-c: Two affine transforms are defined: one for the north ball (dotted circle) and the other for inside solid yellow balls and outside the dashed circle. Elements for affine transform for the rest of the area were interpolated, which are shown in a gray scale from those for the north ball (white) to those for the other parts (black).

transformation. We shall leave the detail of this second scenario to future studies.

\section{B. DAxBPF Algorithm}

We apply DABPF algorithm to compensate for the spaceand time-dependent affine transform $\Gamma_{t, m}$ on a local basis (see Fig. 3). Theoretically, each image pixel $\underline{x}_{t}$ could have different affine transformation $\Gamma_{t, m}\left(\underline{x}_{t}\right), m=1, \ldots, N_{x}^{2}$, where $N_{x}$ is the number of pixels along $(x, y)$-axes. As a pixel-specific compensation is computationally too expensive, we propose the following approach called DAxBPF.

DAxBPF first reconstructs a finite set of images $f_{0, m}\left(\underline{x}_{0}\right)$, $m=1, \ldots, M\left(\ll N_{x}\right)$, at the reference time $t_{0}$ by using DABPF (steps 1-4 in Section II-B) to compensate for $\Gamma_{t, m}$. 
And as Step 5, a weighted summation is then applied to images $f_{0, m}\left(\underline{x}_{0}\right)$ with

$$
f_{0}\left(\underline{x}_{0}\right)=\frac{\sum_{m=1}^{M} W_{0, m}\left(\underline{x}_{0}\right) f_{0, m}\left(\underline{x}_{0}\right)}{\sum_{m=1}^{M} W_{0, m}\left(\underline{x}_{0}\right)}
$$

where $W_{0, m}$ is a spatially varying weight which corresponds to spatially changing elements of affine transformation (Fig. 5). Note that the filtering direction can be independently defined for each ROI.

\section{Data Sufficiency Condition}

Similar to DABPF algorithm, and as Pack et al., empirically stated in [25], the data sufficiency condition for DAxBPF is as follows: a point $\underline{x}_{0}$ at the reference time $t_{0}$ can be reconstructed from fan-beam projections acquired during a time-dependent nonrigid deformation with better quality, provided every line passing through any object point on the filtering line of the point $\underline{x}_{0}$ has an intersection with the virtual source trajectory. The reconstructed point $\underline{x}_{0}$ is with better quality than if the condition is not satisfied. Unfortunately, satisfying the condition in itself does not guarantee that the resulting quality of the image is sufficient.

\section{Evaluation Methods}

Computer simulations were performed to assess the performance of the proposed algorithms in terms of the following aspects with nonrigid motion: 1) overall motion artifact, 2) spatial resolution, 3) nonperiodic motion pattern, 4) the effect of feathering in reconstruction, 5) image noise, 6) tradeoff between image noise and spatial resolution, and 7) off-synchronized motion.

\section{A. General Methods}

We used a 5-ball phantom defined in Table I. Unless otherwise mentioned, the motion of the phantom was as follows: one motion cycle period governed all of balls; the ball $\# 2$ in the north rotated over $60^{\circ}$ about the origin while the others over $30^{\circ}$; all balls contract to 2:1; all balls translate over $(40 \mathrm{~mm}, 20 \mathrm{~mm})$. Specifically, elements of affine transformation in (2) are defined as

$$
\begin{aligned}
A_{t} & =C_{t} \operatorname{Rot}\left(\varphi_{t}\right) \\
C_{t} & =c_{0}+c_{1} \cos \left(\frac{2 \pi t}{T_{R R}}\right) \\
c_{0} & =1.5, \quad c_{1}=-0.5 \\
\varphi_{t} & =\varphi_{0}+\varphi_{1} \cos \left(\frac{2 \pi t}{T_{R R}}\right) \\
B_{i, t} & =B_{i, 1} \sin \left(\frac{2 \pi t}{T_{R R}}\right) ; \quad i=0,1 \\
B_{0,1} & =-20 ; \quad B_{1,1}=-10
\end{aligned}
$$

where $\operatorname{Rot}(\varphi)$ is a rotation matrix for an angle of $\varphi, T_{R R}$ is a time period for one motion cycle, $\left(\varphi_{0}, \varphi_{1}\right)$ for the north ball
TABLE I

Definition of 5-Ball Phantom. Numbers ARE IN MM FOR THE CENTERS AND SIZES AND IN $\mathrm{cm}^{-1}$ FOR LINEAR ATTENUATION COEFFICIENTS $\mu$

\begin{tabular}{ccrrl} 
Index & Center Size & $\mu$ & Note \\
\hline 1 & $(0,0)$ & 200 & 0.182 & muscle \\
2 & $(50,0)$ & 40 & 0.276 & spine (north) \\
3 & $(0,50)$ & 40 & 0.217 & iodine-blood \\
4 & $(-50,0)$ & 40 & 0.175 & fat \\
5 & $(0,-50)$ & 5 & 0.217 & iodine-blood
\end{tabular}

and for the others were $\left(30^{\circ},-30^{\circ}\right)$ and $\left(15^{\circ},-15^{\circ}\right)$, respectively. Three $T_{R R}$ 's that correspond to 60,63 , and 67 cycles-perminute (bpm) were used; their projection ranges were $6 \pi / T_{R R}$, $5.71 \pi / T_{R R}, 5.37 \pi / T_{R R}$, respectively, with a gantry rotation time of $1 / 3 \mathrm{~s} /(2 \pi) \approx 333.3 \mathrm{~ms} / \mathrm{rev}$. At $60 \mathrm{bpm}$, the object motion cycle and projection rotation cycle are in full-sync: $T_{R R}$ corresponds to $6 \pi .72 \mathrm{bpm}$ and $90 \mathrm{bpm}$ are next fully-synchronized conditions, $T_{R R}$ to $5 \pi$ and $4 \pi$, respectively. Taguchi et al. showed that the effect of object motion simply depends on the extent of such synchronization [26]. Thus, studying the motion cycles of 60,63 , and $67 \mathrm{bpm}$ will be sufficient to see the effect of synchronization from a full-sync to a severe off-sync. Among them, we used the motion with $63 \mathrm{bpm}$ intensively.

As the affine elements for the north ball were different from those for the others, we employed the method described in Section III-A and obtained smoothly changing affine transformations (Fig. 5). DABPF used the affine transformation defined for balls \#1, \#3, \#4, and \#5.

Fan-beam projection data were generated over $8 \pi$ with $1 / 3 s /(2 \pi) \approx 333.3 \mathrm{~ms} / \mathrm{rev}$ and monochromatic X-ray at $80 \mathrm{keV}$. The other scan conditions were similar to a clinical X-ray CT scanner: Equiangular 672 samples over $52.14^{\circ} ; 1160$ projections per $2 \pi$; the source-to-isocenter distance of $570 \mathrm{~mm}$. An example of generated projection data is shown in Fig. 5.

Circular images were reconstructed with a matrix of $512^{2}$ over a diameter of 500-mm range. During the image reconstruction with DABPF and DAxBPF methods, when the range of backprojection for an $\underline{x}$ was $n_{\lambda} \pi, n_{\lambda} \in \mathrm{N}^{*}$, a rectangular weight with a height of $1 / n_{\lambda}$ was used for the normalized redundancy weight $w$. When the backprojection range was a fraction of $\pi$, $\left(n_{\lambda}+\beta\right) \pi, 0<\beta<1$, a trapezoidal weight was used whose height was $1 / n_{\lambda}$ in the middle region and linearly reduced over $\beta \pi / 2$ to zero at both edges. For $H^{-1}(\cdot), \underline{\theta}$ was fixed at $(1,0)$, horizontally from left to right; and known pixel value $f_{0}\left(\underline{x}_{0}\right)=0$ for $\left\|\underline{x}_{0}\right\|>220 \mathrm{~mm}$ was used to obtain the offset value (see (17) of [23]). A Shepp-Logan filter [27] and Parker weight [28] was used for FBP method.

The "true images" were obtained as follows. At each time $t$, projection data over $8 \pi$ was obtained without phantom motion; and an image was reconstructed by FBP with a 1/8 weight.

\section{B. Overall Motion Artifact and Spatial Resolution}

The 5-cylinder phantom with a $63 \mathrm{bpm}$ motion was used. Images at $t=0, T_{R R} / 4, T_{R R} / 2$, and $3 T_{R R} / 4$, were reconstructed by FBP, DABPF, and DAxBPF, respectively.

The profiles of the images at $t=0$ and $T_{R R} / 2$ were obtained to assess the accuracy of pixel values and the spatial resolution. 


\section{Backprojection Range and Feathering}

The projection data with $63 \mathrm{bpm}$ was used. In order to evaluate the effect of a nonperiodic object motion, three images were reconstructed by DAxBPF from projections over $2 \pi, 4 \pi$, and $6 \pi$ (corresponding to $0.35,0.70$, and 1.05 cycles). A rectangular redundancy weight was applied in each case.

At the presence of the object motion, a feathering weight is often applied to reduce the effect of inconsistency in projection data [29]-[31]. We assessed the ability of such feathering weights to reduce the motion artifacts. We used a trapezoidal weight and reconstructed images from projections over $2.2 \pi$, $4.2 \pi$, and $6.2 \pi$, respectively, and compared images with a rectangular weight.

\section{Image Noise}

The projection (line integral) data with $63 \mathrm{bpm}$ was converted to the transmitted X-ray intensity with the incident flux of 5000 photons per ray. Poisson noise was then added and the noisy intensity data were then log-converted back to line integrals. Image reconstruction was employed by FBP and DAxBPF with projections over $2.2 \pi, 4.2 \pi$, and $6 \pi$, respectively.

\section{E. Noise-Resolution Tradeoff}

We evaluated the tradeoff between the image noise and spatial resolution at the expanded and contracted phases with and without motion compensation.

We used three projection data under three motion conditions: a) the heart was beating; the heart was stationary either at b) $t=0$ (the expanded phase) or at c) $t=T / 2$ (the contracted phase). One image each at $t=0$ and $T / 2$ was reconstructed using DAxBPF algorithm. DAxBPF reduces to DBPF without motion correction. An index of the spatial resolution was then obtained as follows. A horizontal profile near the north ball was obtained in each image (Fig. 6, arrows); the inverse of the maximum gradient of the profile was obtained and normalized by that of the stationary expanded object $(t=0)$.

Poisson noise was added to projection data using the same procedure as Section IV-D. For each of the three projection sets, we generated 20 realizations of noisy projection data (60 data sets in total). One noisy image each at the expanded and contracted phases was reconstructed from each of noisy data from motion condition a); one noisy image each was reconstructed from each of conditions b) and c). An index of the image noise was then obtained as follows. The standard deviation for each pixel was computed over the 20 noise realizations. These standard deviations were then averaged over the square region of $110 \mathrm{~mm} \times 110 \mathrm{~mm}$ (see Fig. 6) and normalized by the average standard deviation obtained from the stationary expanded object (condition-b). We also computed the standard deviation of standard deviation values over the entire image.

We obtained a tradeoff curve of spatial resolution and noise with Gaussian filter strength as the parameter along the curve: To each image, we applied a $21 \times 21$ Gaussian filter with various strength and measured the above two indexes for noise and spatial resolution.

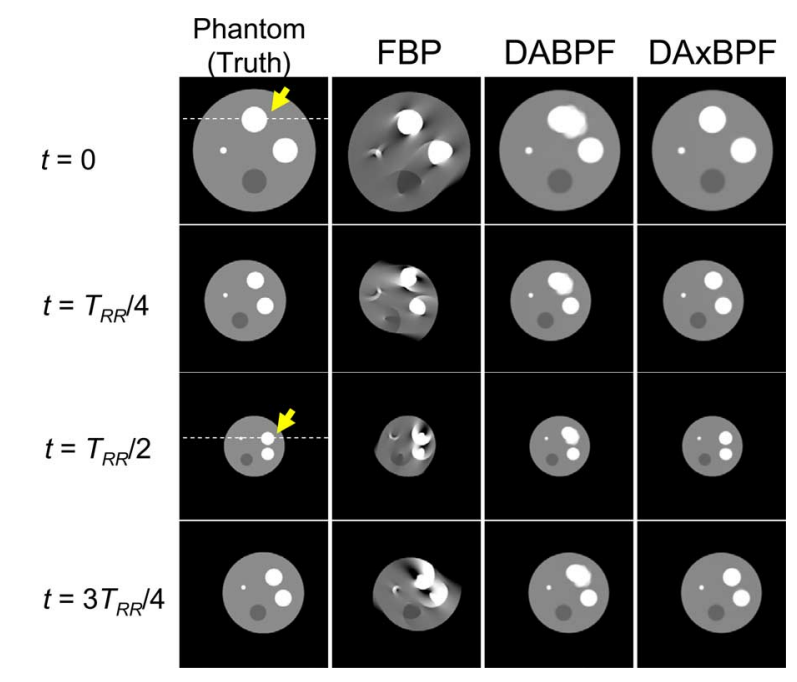

Fig. 6. Images of 5-ball phantom with $63 \mathrm{bpm}$ reconstructed from projections over $1.29 \pi$ (FBP) or $6 \pi$ (DABPF and DAxBPF). Window width/level $(W / L)=0.054 / 0.18$ corresponding to 300 H.U./0 H.U.

\section{F. Off-Synchronized Motion}

We assessed the effect of two kinds of off-synchronizations, inter-off-sync and intra-off-sync, both of which induces an inconsistency between projections at the beginning and the end of backprojection range. In the first experiment, the object motion cycle and the scanner's projection angle were off-sync (inter-off-sync). The cycle of the entire object motion was chosen as 60, 63, and $67 \mathrm{bpm}$ corresponding to a scanner's rotation of $6 \pi /$ cycle (fully synced), $5.71 \pi /$ cycle, $5.37 \pi /$ cycle, respectively. Images were reconstructed from projections over $6 \pi$ using DAxBPF.

In the second experiment, the north ball and the other balls were off-sync (intra-off-sync). The north ball followed 60 and $67 \mathrm{bpm}$ while the others did $63 \mathrm{bpm}$. Images were reconstructed by DAxBPF from data over $6 \pi$ and $6.2 \pi$.

\section{EVAluation Results}

\section{A. Overall Motion Artifact and Spatial Resolution}

Fig. 6 shows images reconstructed by FBP, DABPF, and DAxBPF together with the true phantom images with a narrow window width $\left(0.054 \mathrm{~cm}^{-1}\right.$ or 300 H.U. $)$ to enhance the artifact.

Images of FBP exhibited strong shading/whitening artifact and distorted shape of balls. Images of DABPF showed almost no artifact except for severely smeared north ball. The artifact did not spread out to large area as FBP. Images of DAxBPF presented no visible artifacts in all motion phases.

Horizontal profiles at $t=0$ and $T_{R R} / 2$ shown in Fig. 7 confirmed the above observations: FBP provided shifted or distorted profiles; DABPF also showed distorted profiles; the edge of DAxBPF was slightly blurred at $t=0$ but values in flat regions were accurate.

We made two observations on edge profiles of DAxBPF. First, they were more blurred than that of FBP in general; and second, the sharpness of edges was phase dependent. DAxBPF provided sharper edge profiles at $t=T_{R R} / 2$ (contracted phase) 

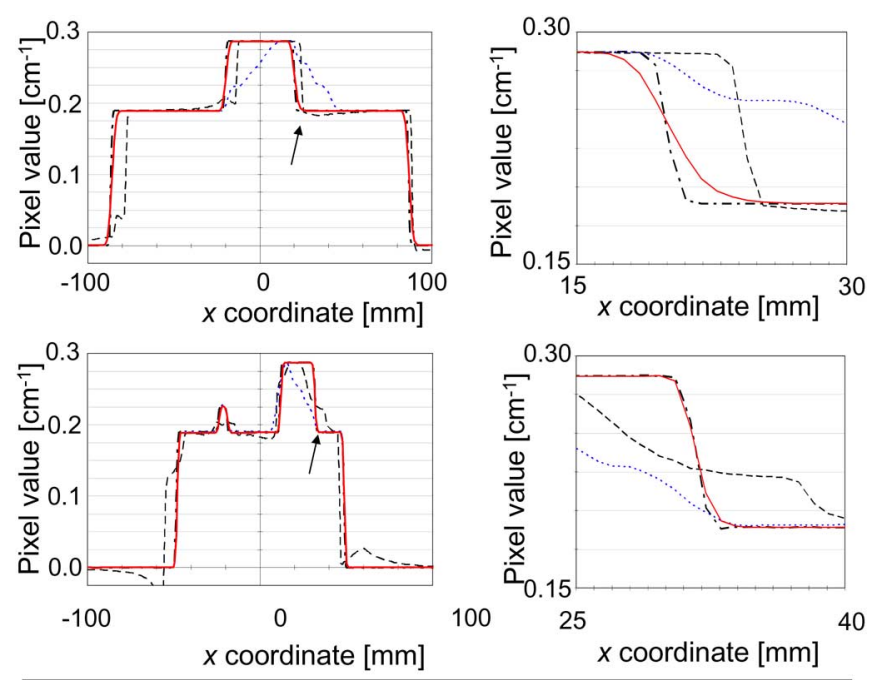

- - - - Phantom (Truth) - - - - FBP

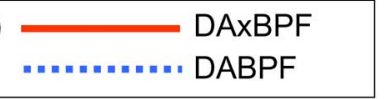

Fig. 7. Horizontal profiles of Fig. 6 indicated at dashed lines at $t=0$ (top) and $T_{R R} / 2$ (bottom); the entire range (left) and the limited range (right) near the edges indicated by arrows.

than those at $t=0$ (expanded phase)—in fact, the profiles at $t=T_{R R} / 2$ are very close to the "truth," FBP without object motion.

We believe common causes of the two phenomena must be the derivative process with respect to $\lambda$ and the motion compensation process. When derivative is calculated by differentiating adjacent samples as in (13) and if those samples are spatially separated in a larger distance than adjacent rays used in the ramp filtering of FBP, the derivative process reduces the spatial resolution. This is a common problem with other methods that use a differentiating step [32]-[34].

The interval of samples is transformed as $n_{0}=A_{t} n_{t}$ during the motion compensation process. The intrinsic Nyquist frequency $f_{N y}$ defined by the sampling intervals along $n_{t}$ at $t$ is then converted to $f_{N y} /\left\|A_{t} n_{t}\right\|$ at the reference time $t_{0}$. The image at the expanded phase $t=0$ is reconstructed with $\left\|A_{t} n_{t}\right\|>1$ for almost all projections, which results in the reduced Nyquist frequency, so as the spatial resolution of images. In contrast, the image at the contracted phase $t=T_{R R} / 2$ is reconstructed with $\left\|A_{t} n_{t}\right\|<1$, thus, shows the improved spatial resolution. If the scale of the object is not isotropic (i.e., scales along $x$ - and $y$ axes are different from each other), it may cause a phase-dependent asymmetric point spread function.

\section{B. Backprojection Range and Feathering}

Fig. 8(a)-(c) shows images reconstructed by DAxBPF from $2 \pi, 4 \pi$, and $6 \pi$, respectively. The effect of nonperiodic motion patterns during the backprojection range was seen as shadings

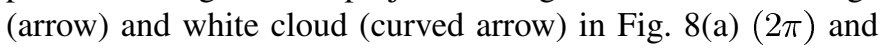
$7 \mathrm{~b}(4 \pi)$. Notice, however, a very narrow window width is used to enhance the shading, which is merely $0.002 \mathrm{~cm}^{-1}$ or a $3.2 \%$ of the difference between the north ball and ball \#1 (on center). The relative rotating motion between the north ball and the rest of the phantom over $2 \pi$ was $21^{\circ}$, which was twice as large as the rotating motion over $\pi\left(10.5^{\circ}\right)$. Comparing Fig. 8(a) with the
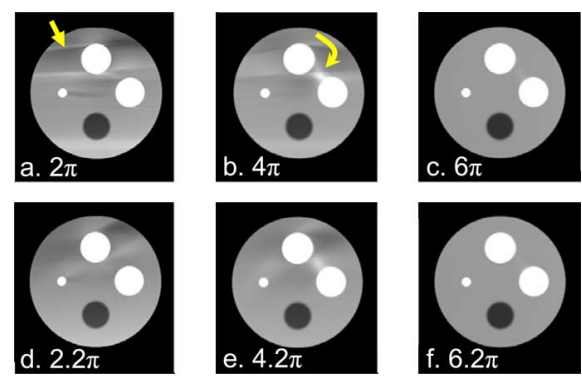

Fig. 8. Images reconstructed with various angular ranges. $W / L=$ $0.018 / 0.18$ corresponding to 100 H.U./0 H.U.; the narrow window is used to visualize the artifact. Smaller ranges show stronger artifacts $(a-b)$, which are reduced by using a trapezoidal feathering weight (d-e).
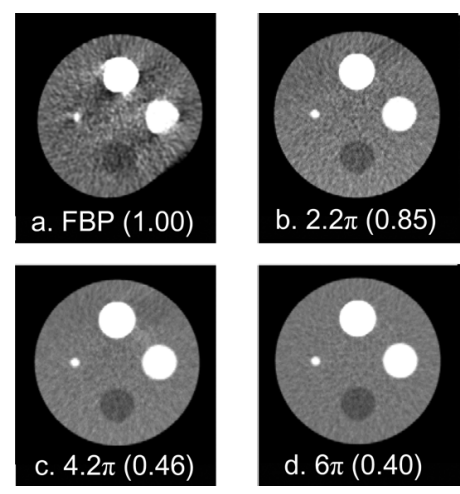

Fig. 9. Images reconstructed from noisy projections. $W / L=0.054 / 0.18$. Image noise decreases as increasing the projection angular range $(b \rightarrow c \rightarrow d)$.

FBP in Fig. 6, we found the proposed local correction worked noticeably well to reduce the effect of nonrigid motion.

The visibility of the shading artifact was significantly reduced by using a slight overlap $(0.2 \pi)$ with a trapezoidal feathering weight [Fig. 8(d)-(f)]—although the reduced pixel value remained.

\section{Image Noise}

Fig. 9 shows reconstructed noisy images at $t=0$. In order to compare image noise with the same point spread function, a $7 \times 7$ Gaussian filter was applied to the image reconstructed by FBP to produce Fig. 9(a). A square ROI was used at the isocenter to measure the standard deviation of pixel values, which was normalized against that of filtered FBP image. The normalized standard deviation value of DAxBPF method decreased with increasing backprojection range: 0.85 with $2.2 \pi, 0.46$ with $4.2 \pi$, and 0.40 with $6 \pi$, respectively. These results generally agree with a simple theoretical prediction by the amount of effective photons contributed to images: $(\pi / 2 \pi)^{1 / 2}=0.71$, $(\pi / 4 \pi)^{1 / 2}=0.50$, and $(\pi / 6 \pi)^{1 / 2}=0.41$.

\section{Noise-Resolution Tradeoff}

Fig. 10(b) shows standard deviation values computed over 20 noise realizations at $t=0$ with the beating phantom. The standard deviation reduced to 1.7:1 with all of four cases with increasing a distance from the center from 0 to $100 \mathrm{~mm}$. There was no angular dependency.

Fig. 10(a) shows four tradeoff curves. Four observations were made: 1) when the object was stationary, images at the 


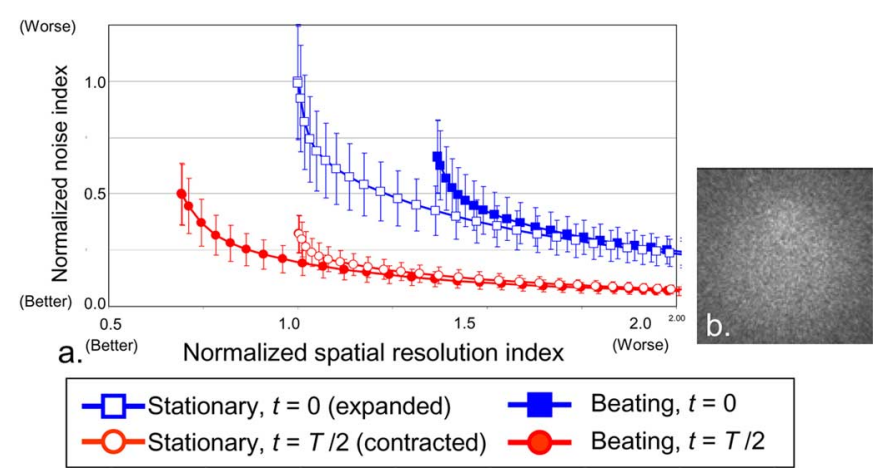

Fig. 10. a: Noise-resolution tradeoff curves. b: Standard deviation map obtained from the beating heart at $t=0$. Motion compensation process of the beating object introduces the motion phase-dependent spatial resolution.

two phases had different noise level at the same spatial resolution; 2) when the object was beating, images at the two phases had different spatial resolutions; 3 ) at the expanded phase, the tradeoff curve for the beating phantom is inferior to that for the stationary phantom; and 4) at the contracted phase, the tradeoff curve for the beating phantom indicates a better performance than for the stationary phantom.

The first observation is attributed to the difference in phantom size. Larger objects attenuate X-ray photons more, and produce noisier projection data (and reconstructed images) for a given incident X-ray photon flux. And discussion in Section V-A explains the mechanism of the second observation.

A further study is required to understand the nature of the tradeoffs, observations 3 and 4, in detail. However, we think the logarithm conversion process may be the major cause. Photon noise in acquired X-ray intensity data is Poisson distributed in this study (it will be compound Poisson with additive electronic noise in actual data), which is log-converted for line integrals and then scaled during the motion compensation process of DAxBPF. This nonlinear process may introduce a favorable tradeoff for DAxBPF when images at the contracted phase were reconstructed. Since the gain/loss of tradeoff curves must depend on the shape and the motion of the object, we cannot definitively conclude that this property is or is not an advantage of DAxBPF when applied to clinical patient data.

\section{E. Off-Synchronized Motion}

Fig. 11 shows difference images with and without inter-synchronization from the image of FBP with a stationary object at $t=0$. The motion cycle of the entire object and the rotation cycle of the scanner were in full-sync (a: $60 \mathrm{bpm}$ ), moderately off-sync (b: $63 \mathrm{bpm}$ ), and largely off-sync (c: $67 \mathrm{bpm})$. Both images with out-of-sync were almost identical to a fully-synchronized case $(60 \mathrm{bpm})$, demonstrating the robustness of DAxBPF algorithm.

Fig. 12 presents difference images with intra-off-sync from the image of FBP with the stationary object. A negligible level of derivation of pixel values $\left( \pm 0.0002 \mathrm{~cm}^{-1}, 1 \mathrm{H} . \mathrm{U} ., \pm 0.1 \%\right)$ was observed (arrows), which was in fact reduced by applying the feathering.

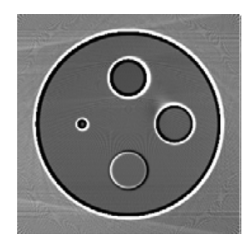

a. $60 \mathrm{bpm}, 6 \pi$

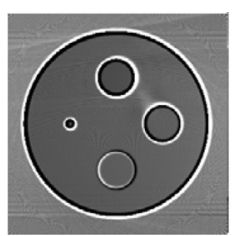

b. $63 \mathrm{bpm}, 6 \pi$

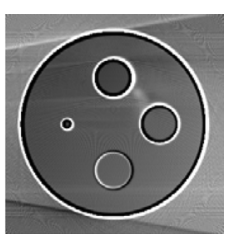

c. $67 \mathrm{bpm}, 6 \pi$
Fig. 11. Difference images of inter-off-synchronization from the image FBP with a stationary object at $t=0 . W / L=0.0054 / 0$ corresponding to 30 H.U./0 H.U. An extremely narrow window is used to demonstrate the differences are very small.

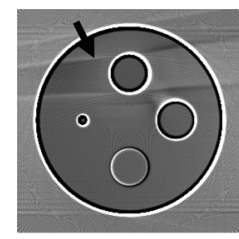

a. $60 \mathrm{bpm}, 6 \pi$

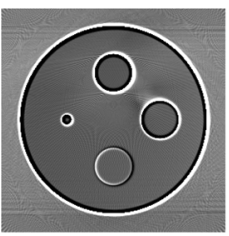

c. $60 \mathrm{bpm}, 6.2 \pi$

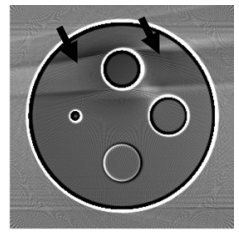

b. $67 \mathrm{bpm}, 6 \pi$

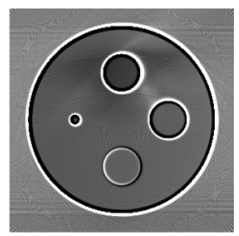

d. $67 \mathrm{bpm}, 6.2 \pi$
Fig. 12. Difference images of intra-off-synchronization from the image of FBP with a stationary object at $t=0 . W / L=0.054 / 0$ corresponding to 30 H.U./0 H.U. An extremely narrow window is used to visualize artifacts (a-b, arrows) which are reduced by a feathering $(\mathrm{c}-\mathrm{d})$.

\section{DISCUSSION AND CONCLUSION}

We developed an exact DABPF algorithm that compensates for a time-dependent affine transformation of the object. We also developed an approximate algorithm, DAxBPF, to reduce the effect of norigid motion of the object. The results showed that an approximate DAxBPF algorithm, which applies DABPF on a local basis, reconstructs images with good quality. DAxBPF significantly reduces the motion artifact compared with the current cardiac algorithm (FBP with Parker weight). The proposed DAxBPF also reduces the image noise when it uses projections over a larger range than Parker weight does. A $60 \%$ noise reduction from the current level, if directly converted, implicates an $83 \%$ dose reduction from the current level (from $10-15 \mathrm{mSv}$ to $1.7-2.6 \mathrm{mSv}$ ) to obtain images with the current noise level. This could have a substantial impact on the public health.

Our results show that the reconstruction from projections over $2 \pi$ is likely to be acceptable; yet images over $6 \pi$ or quasi-one cycle is better in terms of the intensity of artifacts and image noise. The use of data over one heart cycle is practically possible in clinical settings. The current cardiac CT protocols choose a helical pitch between $0.2-0.33$ (corresponding to $6 \pi-10 \pi$ for each point $\underline{x}$ ) depending on the patient heart rate, such that each point $\underline{x}$ is covered at least over one heart cycle.

The affine motion model does not preserve the mass of the object as it deforms. It proportionally changes the mass with the scale. This motion model may not be a right one for various 
motions of human body. However, as the heart expands and contracts, iodine-mixed blood comes into and goes out of chambers (e.g., left ventricle), which drastically increases and decreases the total mass. Thus, in fact, affine transform is a better model for cardiac imaging than those which preserve the mass.

A natural question one may ask is, "Why does DAxBPF work well for nonrigid motion?" Unfortunately, we have been unsuccessful in providing a mathematical explanation. Intuitive reasoning is a slow and quasi-periodic object motion relative to the projection angle. Let us consider an object which consists of a finite set of delta functions and assume a nonrigid motion simply moves their locations, $f(\underline{x})=\Sigma_{m} \delta\left(\underline{x}_{m}\right)$, where $m$ is an index for delta functions. The filtered parallel projection data can then be decomposed into a finite set of sinograms. With nonrigid motion, the backprojection (integration) process introduces an error at a point $\underline{x}$ as each sinogram is radially shifted due to the motion of the delta function at $\underline{x}_{m, t}$, for each time $t$. The effect of such shift introduces inaccurate values, weights and integration range. If the motion within the integration range is drastic, the effect is significant. However, if the motion is slow and smooth within the angular range, the error remains small. A quasi-periodic motion over the angular range also reduces the inconsistency between the beginning and the end of angular range, thus, reduces the artifact. We tested phantoms with various off-sync and nonperiodic motions reasonable in cardiac imaging (Figs. 6, 8 , and 9) and obtained good results.

The above intuitive explanation seems applicable to other analytical reconstruction algorithms with different filtering kernels. For example, applying the local compensation approach to Roux's and Desbat's DAFBP algorithms [18], [20], [21] would yield DAxFBP algorithm. It would be interesting to study whether such DAxFBP provides as good images as DAxBPF. We plan to implement DAxFBP and evaluate its performance.

The proposed DAxBPF will provide good images even at the presence of transverse truncation as DBPF does [23], [24]. As DAxBPF do not require projection data outside of the region-ofinterest and its filtering lines, it also has a potential to reduce necessary radiation dose to the patient in cardiac scan.

The disadvantage of DAxBPF is a computational expense. The current FBP requires $O\left(N^{3}\right)$ calculations for the ramp filtering process and $O\left(N^{3}\right)$ calculations for backprojection process. If $M$ sets of motion models are used along each filtering line, DAxBPF will first reconstruct $M$ images, and then apply a weighted summation. Thus, DAxBPF requires $O\left(M N^{3}\right)$ for finite inverse Hilbert transform and $O\left(M N^{3}\right)$ for backprojection process. An acceleration process such as fast Fourier transform is not applicable for finite inverse Hilbert transform. Therefore, DAxBPF algorithm will be approximately $M$ times computationally demanding than the current FBP methods. The appropriate number of motion models in one dimension, $M$, depends on the complexity of the motion, thus, is of interest in future works. From literatures for modeling and estimation of cardiac motion [10]-[16], we assume 10-30 would be sufficient to cover the human heart $(100 \mathrm{~mm})$ with which the nonrigid motion over $3-10 \mathrm{~mm}$ range is approximated by one affine motion model.

Future works are as follows. The image quality with different nonrigid motion patterns needs to be further evaluated. The

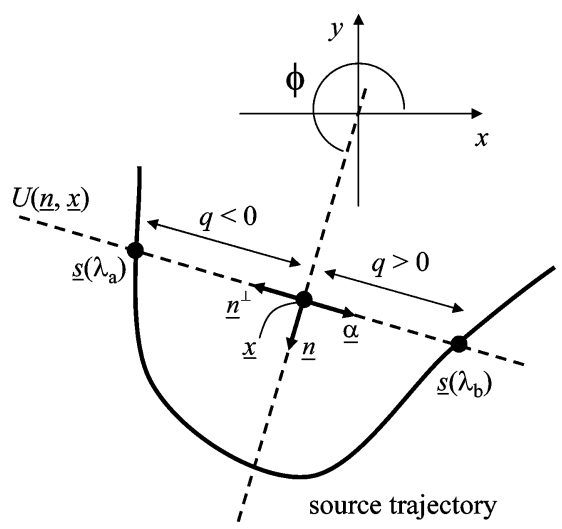

Fig. 13. Line $U$ passes through a point $\underline{x}$ and sources at $\underline{s}\left(\lambda_{a}\right)$ and $\underline{s}\left(\lambda_{b}\right)$.

phantom we used in this study was not too simple; however, as the algorithm remains approximate, one has to carefully examine the performance of DAxBPF method with more complex motions, such as 4-D NCAT phantom or clinical cardiac data.

The spatial resolution of image needs to be improved. We believe, as discussed in Section V-A, one of its major causes is the derivative (or differentiation) step with respect to $\lambda$. One can use a new scheme recently proposed by Noo et al. [35] with a chain rule: it does not require a significant change from the current formulae. Another solution is to use integration by parts as Zou et al. did in [36], which, however, requires a significant change of reconstruction formulae. Moreover, there is a concern as we expect an increased spatial variation of image noise as $\left\|\underline{x}_{0}-\underline{s}_{0}(\lambda)\right\|^{-2}$ weight will replace $\left\|\underline{x}_{0}-\underline{s}_{0}(\lambda)\right\|^{-1}$ weight.

The extension of the proposed fan-beam DAxBPF method to cone-beam reconstruction is relatively straightforward. The derivative of cone-beam projections is taken with respect to the source parameter, $\lambda$, followed by a weighted cone-beam backprojection and a 1-D finite inverse Hilbert transform. The optimal filtering direction of 1-D Hilbert transform is an interest of the research. Candidates include an actual PI-line formed by virtual trajectory and a virtual PI-line [37] that does not connect two source points in either virtual or actual trajectories.

In conclusion, we have developed fan-beam reconstruction algorithms using derivative backprojection filtering approach to compensate for a time-dependent deformation. DABPF method for a global affine transformation is exact. DAxBPF for a nonrigid transformation are approximate, which showed promising results with computer simulations.

\section{APPENDIX}

For the sake of completeness, we rederive with consistent notations fan-beam DBPF algorithm for an arbitrary orbit.

In the reference time or for the stationary object, parallelbeam projection can be expressed as

$$
p(r, \phi)=\int_{-\infty}^{\infty} f\left(r \underline{n}+r^{\prime} \underline{n}^{\perp}\right) d r^{\prime}
$$

where $\underline{n}=(\cos \phi, \sin \phi)$, and $\underline{n}^{\perp}=(-\sin \phi, \cos \phi)$ (see Fig. 13). 
DBPF algorithm for a parallel beam projections [23] can be written as

$$
\begin{aligned}
p(r, \phi) & =\lim _{\varepsilon \rightarrow 0} \frac{p(r+\varepsilon, \phi)-p(r, \phi)}{\varepsilon} \\
b(\underline{x}) & =\int_{\phi_{1}}^{\phi_{2}} w(r, \phi) \operatorname{sgn}(\underline{\theta} \cdot \underline{n}) p^{\prime}(r, \phi) d \phi \\
f(\underline{x}) & =-\frac{1}{2 \pi} H^{-1}(b(\underline{x}))
\end{aligned}
$$

where $H^{-1}(\cdot)$ is the 1-D finite inverse Hilbert transform along $\underline{\theta}$ direction [23] and $w$ is a normalized redundancy weight that satisfies

$$
\sum_{i=0}^{\infty}[w(r, \phi \pm 2 i \pi)+w(-r, \phi \pm(2 i+1) \pi)]=1 .
$$

Parallel- and fan-beam projections are related by

$$
p(r, \phi)=\left.g(\lambda, \underline{\alpha})\right|_{\underline{s}}(\lambda) \cdot \underline{n}=r, \underline{\alpha} \cdot \underline{n}=0 .
$$

Taking a derivative with respect to $\lambda$ of both sides of (29) yields

$$
p^{\prime}(r, \phi)=\left.\frac{g^{\prime}(\lambda, \underline{\alpha})}{\underline{s}^{\prime}(\lambda) \cdot \underline{n}}\right|_{\underline{s}(\lambda) \cdot \underline{n}=r, \underline{\alpha} \cdot \underline{n}=0} .
$$

For simplicity, we use a prime sign to indicate the derivative with respect to $\lambda$ (e.g., $g^{\prime} \equiv \partial g / \partial \lambda$ ). This rebinning formula (30) can only hold for nonzero $\underline{s}^{\prime}(\lambda) \cdot \underline{n}$, thus, the tangent vector $\underline{s}^{\prime}(\lambda)$ must not be orthogonal to $\underline{n}$. Any source points $\underline{s}\left(\lambda_{a}\right)$ and $\underline{s}\left(\lambda_{b}\right)$ on the line $U$ can be parameterized by a distance $q$

$$
\underline{x}+q \underline{n}^{\perp}=\underline{s}(\lambda)
$$

where $|q|=\|\underline{x}-\underline{s}(\lambda)\|$. Differentiating each side of (31) with respect to $\lambda$ yields

$$
\frac{d q}{d \lambda} \underline{n}^{\perp}+q \underline{n} \frac{d \phi}{d \lambda}=\underline{s}^{\prime}(\lambda) .
$$

Thus

$$
\underline{s}^{\prime}(\lambda) \cdot \underline{n}=q \frac{d \phi}{d \lambda} \text { and } \frac{d \phi}{d \lambda}=\frac{\underline{s}^{\prime}(\lambda) \cdot \underline{n}}{\|\underline{x}-\underline{s}(\lambda)\|} .
$$

Inserting (30) into (25) and (33) into (26) yields a fan-beam BPF formulae for an arbitrary source orbit

$$
\begin{aligned}
g^{\prime}(\lambda, \bar{\alpha}) & =\lim _{\varepsilon \rightarrow 0} \frac{g(\lambda+\varepsilon, \underline{\alpha})-g(\lambda, \underline{\alpha})}{\varepsilon} \\
b_{\underline{\theta}}(\underline{x}) & =\int_{\lambda_{1}}^{\lambda_{2}} \frac{w(\underline{s} \cdot \underline{n}, \underline{n}) \operatorname{sgn}(\underline{\theta} \cdot \underline{n}) g^{\prime}(\lambda, \underline{\alpha})}{\|\underline{x}-\underline{s}\|} d \lambda
\end{aligned}
$$

where

$$
\begin{aligned}
& \underline{n}=\underline{s^{\prime}}-\left[\underline{\alpha} \cdot \underline{s}^{\prime}\right] \cdot \underline{\alpha} \\
& \underline{\alpha}=\frac{(\underline{x}-\underline{s})}{\|\underline{x}-\underline{s}\|} .
\end{aligned}
$$

and the sum of the redundancy weights to rays from $\lambda_{i}$ that belong to the same line passing through a point $\underline{x}$ is 1

$$
\sum_{i=0}^{\infty} w(\underline{s} \cdot \underline{n}, \underline{n})=1 \text {. }
$$

\section{ACKNOWLEDGMENT}

The authors would like to thank W. P. Segars for his help with CT simulation program, and F. Noo, T. Flohr, G. Lauritsch, and J. Xu for their discussion.

\section{REFERENCES}

[1] The Burden of Chronic Diseases and Their Risk Factors: National and State Perspectives. Atlanta, GA: National Center for Chronic Disease Prevention and Health Promotion, 2004.

[2] M. Gilard, J.-C. Cornily, P.-Y. Pennec, C. Joret, G. L. Gal, J. Mansourati, J.-J. Blanc, and J. Boschat, "Accuracy of multislice computed tomography in the preoperative assessment of coronary disease in patients with aortic valve stenosis," J. Amer. College Cardiol., vol. 47, pp. 2020-2024, 2006.

[3] K. Taguchi, W. P. Segars, G. S. K. Fung, and B. M. W. Tsui, "Toward time resolved 4-D cardiac CT imaging with patient dose reduction: Estimating the global heart motion," in Med. Imag. 2006: Phys. Med. Imag.. San Diego, CA: , 2006, pp. 61420J-9.

[4] J. Hsieh, J. Londt, M. Vass, J. Li, X. Tang, and D. Okerlund, "Step-andshoot data acquisition and reconstruction for cardiac X-ray computed tomography," Med. Phys., vol. 33, pp. 4236-4248, 2006.

[5] P. J. L. Riviere, "Penalized-likelihood sinogram smoothing for lowdose CT," Med. Phys., vol. 32, pp. 1676-1683, 2005.

[6] P. J. L. Riviere, B. Junguo, and P. A. Vargas, "Penalized-likelihood sinogram restoration for computed tomography," IEEE Trans. Med. Imag., vol. 25, no. 8, pp. 1022-1036, Aug. 2006.

[7] J. Hsieh, "Adaptive streak artifact reduction in computed tomography resulting from excessive X-ray photon noise," Med. Phys., vol. 25, pp. 2139-2147, 1998.

[8] D. R. Gilland, B. A. Mair, J. E. Bowsher, and R. J. Jaszczak, "Simultaneous reconstruction and motion estimation for gated cardiac ECT," IEEE Trans. Nucl. Sci., vol. 49, no. 5, pp. 2344-2349, Oct. 2002.

[9] D. R. Gilland, B. A. Mair, and J. Sun, "Joing 4-D reconstruction and motion estimation in gated cardiac ECT," in 8th Int. Conf. Fully Threedimensional Reconstruction Radiol. Nucl. Med., Salt Lake City, UT, 2005, pp. 303-306.

[10] S. M. Song and R. M. Leahy, "Computation of 3-D velocity fields from 3-D cine CT images of a human heart," IEEE Trans. Med. Imag., vol. 10, no. 3, pp. 295-306, Sep. 1991.

[11] B. K. P. Horn and B. G. Schunck, "Determining optical flow," Artif. Intell., vol. 17, pp. 185-203, 1981.

[12] G. J. Klein, B. W. Reutter, and R. H. Huesman, "Non-rigid summing of gated PET via optical flow," IEEE Trans. Nucl. Sci., vol. 44, no. 4, pp. 1509-1512, Aug. 1997.

[13] R. Zeng, J. A. Fessler, and J. M. Balter, "Respiratory motion estimation from slowly rotating X-ray projections: Theory and simulation," Med. Phys., vol. 32, pp. 984-991, 2005.

[14] R. Zeng, J. A. Fessler, and J. M. Balter, "Estimating 3-D respiratory motion from orbiting views by tomographic image registration," IEEE Trans. Med. Imag., vol. 26, no. 2, pp. 153-163, Feb. 2007.

[15] M. Wierzbicki, G. M. Guiraudon, D. L. Jones, and T. Peters, "Dose reduction for cardiac CT using a registration-based approach," Med. Phys., vol. 34, pp. 1884-1895, 2007.

[16] D. Shen, H. Sundar, Z. Xue, Y. Fan, and H. Litt, "Consistent estimation of cardiac motions by 4-D image registration," in Medical Image Computing and Computer-Assisted Intervention. Heidelberg, Germany: Springer, 2005, pp. 902-910.

[17] C. R. Crawford, K. F. King, C. J. Ritchie, and J. D. Godwin, "Respiratory compensation in projection imaging using a magnification and displacement model," IEEE Trans. Med. Imag., vol. 15, no. 3, pp. 327-332, Jun. 1996.

[18] S. Roux, L. Desbat, A. Koenig, and P. Grangeat, "Exact reconstruction in 2-D dynamic CT: Compensation of time-dependent affine deformations," Phys. Med. Biol., vol. 49, pp. 2169-2182, 2004.

[19] F. Noo, M. Defrise, R. Clackdoyle, and H. Kudo, "Image reconstruction from fan-beam projections on less than a short scan," Phys. Med. Biol., vol. 47, pp. 2525-2546, 2002.

[20] L. Desbat, S. Roux, and P. Grangeat, "Compensation of some time dependent deformations in 2-D tomography," in 8th Int. Meeting Fully Three-Dimensional Image Reconstruction Radiol. Nucl. Med., Salt Lake City, UT, 2005, pp. 129-132.

[21] L. Desbat, S. Roux, and P. Grangeat, "Compensation of some time dependent deformations in tomography," IEEE Trans. Med. Imag., vol. 26, no. 2, pp. 261-269, Feb. 2007. 
[22] C. J. Ritchie, C. R. Crawford, J. D. Godwin, K. F. King, and K. Yongmin, "Correction of computed tomography motion artifacts using pixel-specific back-projection," IEEE Trans. Med. Imag., vol. 15, no. 3, pp. 333-342, Jun. 1996.

[23] F. Noo, R. Clackdoyle, and J. D. Pack, "A two-step Hilbert transform method for 2-D image reconstruction," Phys. Med. Biol., vol. 49, pp. 3903-3923, 2004.

[24] Y. Zou and X. Pan, "Exact image reconstruction on PI-lines from minimum data in helical cone-beam CT," Phys. Med. Biol., vol. 49, pp. 941-959, 2004.

[25] J. D. Pack and F. Noo, "Dynamic computed tomography with known motion field," in Medical Imag. 2004: Image Process.. San Diego, CA: , 2004, pp. 2097-2104.

[26] K. Taguchi, B. S. Chiang, and I. A. Hein, "Direct cone-beam cardiac reconstruction algorithm with cardiac banding artifact correction," Med. Phys., vol. 33, pp. 521-539, 2006.

[27] A. C. Kak and M. Slaney, Principles of Computerized Tomographic Imaging. Piscataway, NJ: IEEE Press, 1987.

[28] D. L. Parker, "Optimal short scan convolution reconstruction for fanbeam CT," Med. Phys., vol. 9, pp. 254-7, Mar.-Apr. 1982.

[29] C. R. Crawford and K. F. King, "Computed tomography scanning with simultaneous patient translation," Med. Phys., vol. 17, pp. 967-982, 1990.

[30] K. Taguchi, "Temporal resolution and the evaluation of candidate algorithms for four-dimensional CT," Med. Phys., vol. 30, pp. 640-50, Apr. 2003.
[31] K. Taguchi and H. Anno, "High temporal resolution for multislice helical computed tomography," Med. Phys., vol. 27, pp. 861-72, May 2000.

[32] A. Kastevich, "Analysis of an exact inversion algorithm for spiral conebeam CT," Phys. Med. Biol., vol. 47, pp. 2583-2597, Jul. 2002.

[33] A. Katsevich, "Theoretically exact filtered backprojection-type inversion algorithm for spiral CT," SIAM J. Appl. Math., vol. 62, pp. 2012-2026, Jul. 2002.

[34] A. Katsevich, K. Taguchi, and A. A. Zamyatin, "Formulation of four katsevich algorithms in native geometry," IEEE Trans. Med. Imag., vol. 25, no. 7, pp. 855-868, Jul. 2006.

[35] F. Noo, S. Hoppe, F. Dennerlein, G. Lauritsch, and J. Hornegger, "A new scheme for view-dependent data differentiation in fan-beam and cone-beam computed tomography," Phys. Med. Biol., vol. 52, pp. 5393-5414, 2007.

[36] Y. Zou and X. Pan, "Image reconstruction on PI-lines by use of filtered backprojection in helical cone-beam CT," Phys. Med. Biol., vol. 49, pp. 2717-2731, 2004.

[37] X. Pan, D. Xia, Y. Zou, and L. Yu, "A unified analysis of FBP-based algorithms in helical cone-beam and circular cone- and fan-beam scans," Phys. Med. Biol., vol. 49, pp. 4349-4369, 2004. 